\title{
Effect of doping profile and the work function variation on performance of double-gate TFET
}

\author{
Muhammad Elgamal ${ }^{1, *}$, Aya Sinjab ${ }^{1}$, Mostafa Fedawy ${ }^{1}$, Ahmed Shaker $^{2}$ \\ ${ }^{1}$ Arab Academy for Science, Technology and Maritime Transport, \\ Heliopolis, Cairo, 11799, EGYPT \\ ${ }^{2}$ Ain Shams University, \\ Abbaseyya, Cairo, 11535, EGYPT \\ *Corresponding Author
}

DOI: https://doi.org/10.30880/ijie.2019.11.07.006

Received 30 May 2019; Accepted 30 July 2019; Available online 10 August 2019

\begin{abstract}
Abstract Tunnel Field Effect Transistor (TFET) can be considered as one of the promising transistors because it can switch ON and OFF at lower voltages than the operation voltage of the metal oxide semiconductor field effect transistor (MOSFET). This paper presents the effects of gate electrode work function and the doping profile terminating within and outside the drain on the ambipolar current, the ION/IOFF ratio, and the subthreshold swing. The results show that, Gaussian doping profile terminating within the drain is the most promising for on/off ratio. All the simulations and results have been performed and obtained with the help of ATLAS device simulator (Silvaco) and MATLAB.
\end{abstract}

Keywords: Drain Work Function, Uniform doping, Gaussian doping, On current, Ambipolar current and Subthreshold Swing.

\section{Introduction}

As we scale down the transistor the power consumption of the device decreases [1]. As devices with channel lengths like $90 \mathrm{~nm}$ or above are useful for non-digital applications [2], the general trend in digital world is to scale down the device, and below certain limit, short channel effects prevail [1]. Several alternative devices that resists short channel performance exist.

The Tunnel Field Effect Transistors (TFET) is identified as a reverse-biased p-i-n structure. Unlike the current in a conventional MOSFET it is not based on thermionic emission of carriers but the tunneling of these carriers through a potential barrier. The tunneling probability is high when the tunnel barrier is thin and the difference in the potential barrier height and the particle energy is small. As a result, keeping the power consumption within an acceptable limit is easily achieved feasible. The TFETs, due to their different mechanism of current transport from MOSFET exhibit several interesting electrical characteristics such as the potential for lowering the $60 \mathrm{mV} /$ decade subthreshold swing, ultra-low power consumption, ultra-low voltage operation, better short-channel performance, reduction in the leakage currents, exceeding the speed requirements due to tunneling effects, ability to work on sub-threshold and superthreshold voltage with similarity in fabrication process as compared to MOSFET and higher IOFF/ION current ratio. In this work, we choose a (DG-TFET) structure, as shown in Fig. 1. The list of parameters used is given in Table 1. This paper has been organized as follows. In section 2, the working principles and device physics of DG-TFET is presented. The simulation results are discussed in section 3. Finally, the conclusion is presented in section 4 . 



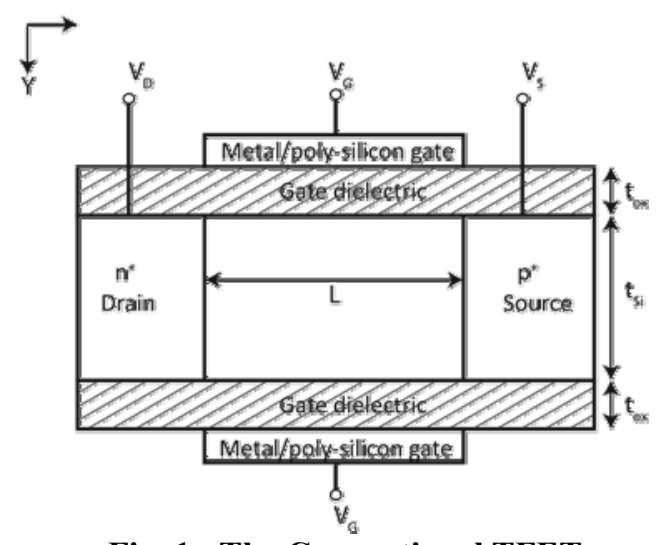

Fig. 1 - The Conventional TFET

Table 1 - The device parameters

\begin{tabular}{lc}
\hline The device parameter & Value/Range \\
\hline Source Doping $\left(\mathrm{N}_{\mathrm{A}}\right)$ & $1 \times 10^{20}$ atoms $/ \mathrm{cm}^{3}$ \\
Drain Doping $\left(\mathrm{N}_{\mathrm{D}}\right)$ & $5 \times 10^{18}, 1 \times 10^{19}, 5 \times 10^{19} \mathrm{atoms} / \mathrm{cm}^{3}$ \\
Channel Doping $\left(\mathrm{N}_{\mathrm{A}}\right.$ or $\left.\mathrm{N}_{\mathrm{D}}\right)$ & $1 \times 10^{17}$ atoms $/ \mathrm{cm}^{3}$ \\
Source Length & $60 \mathrm{~nm}$ \\
Drain Length & $60 \mathrm{~nm}$ \\
Channel Length $(\mathrm{L})$ & $50 \mathrm{~nm}$ \\
Gate Oxide Thickness $\left(\mathrm{t}_{\mathrm{ox}}\right)$ & $3 \mathrm{~nm}$ \\
Silicon Body Thickness $\left(\mathrm{t}_{\mathrm{si}}\right)$ & $10 \mathrm{~nm}$ \\
Gate Work Function $\left(\Phi_{\mathrm{m}}\right)$ & $3.8 \mathrm{eV} 4.5 \mathrm{eV}$ \\
\hline
\end{tabular}

\section{Working principles and device physics}

The work mechanism of a TFET is based on band-to-band tunneling. BTBT employs tunneling of carriers from the valence band into the conduction band through the forbidden bandgap or vice versa. Generating current in both the on and off state which are the Ion where the VGS $>$ VT and IOFF where VGS $<$ VT. IOFF has $\mathrm{I}_{\text {thermal }}$ that decreases by increasing the bandgap of the TFET material. As the gate voltage increase the drain current increases which is quantified by the subthreshold swing.

The subthreshold swing is defined as the change required for increasing the drain current by a factor of 10 in the gate voltage. A low subthreshold swing is always targeted, for low power consumption by gate voltage that controls the on current. As for the other on region responsible the threshold voltage which can be controlled by the work function of the gate material. The threshold voltage has a linear relationship with the work function of the gate [3]. The threshold voltage is defined as the gate voltage at which the drain current reaches $1 \times 10-7 \mathrm{~A}$ at VDS equal to the supply voltage which is taken to be one volt.

The work function of the gate controls the height of the potential barrier which predominate the electrons energy making the tunneling probability that is calculated by Eq. (1) decrease. Another factor that controls the probability of tunneling is the width of the barrier which is controlled by the doping whether it was doped with acceptors or donors to provide available states in addition to the width of the channel exponentially related to the tunneling probability. If the tunneling width is decreased, the tunneling probability increases exponentially [3].

$$
P_{t}=\exp \left[-\frac{4 \sqrt{2 m}}{3 \hbar F}\left(U_{0}-E\right)^{2} \frac{8}{2}\right]
$$

$P_{t}$ is the probability of tunneling, $F$ is the electric field, $m$ is the mass of the particle, and $h$ is the Planck constant, $\left(U_{0}-\right.$ $E)$ is the difference between the height of the barrier and the energy of the particle [5]. In order to obtain the results, the 
following Silvaco Atlas models have been used: bandgap narrowing, Shockley-Read-Hall (SRH) recombination using concentration-dependent lifetimes, concentration-dependent mobility, field-dependent mobility, non-local quantum barrier tunneling model, the tunneling current direction, the conduction band edge, the valence band edge and temperature-dependent band parameters.

\section{Results and discussion}

Data sets were generated from Silvaco simulator and then analyzed via MATLAB to extract different parameters such as on current, ambipolar current, subthreshold swing and on/off ratio. Besides, other parameters that were used to understand these parameters. In addition to comparing them and generating the following graphs.

The use of different doping concentrations $5 \times 10^{18}$ atoms $/ \mathrm{cm}^{3}$ and $1 \times 10^{19}$ atoms $/ \mathrm{cm}^{3}$ will be studied in different doping profiles as these shown in Fig. 2. Specifically, the uniform doping profile, Gaussian profile terminating within the $60-\mathrm{nm}$ drain, and other Gaussian profile terminating deeper in the channel will be studied as doping profiles. The performance of the device will be investigated through the change of work function of the both gates [3].

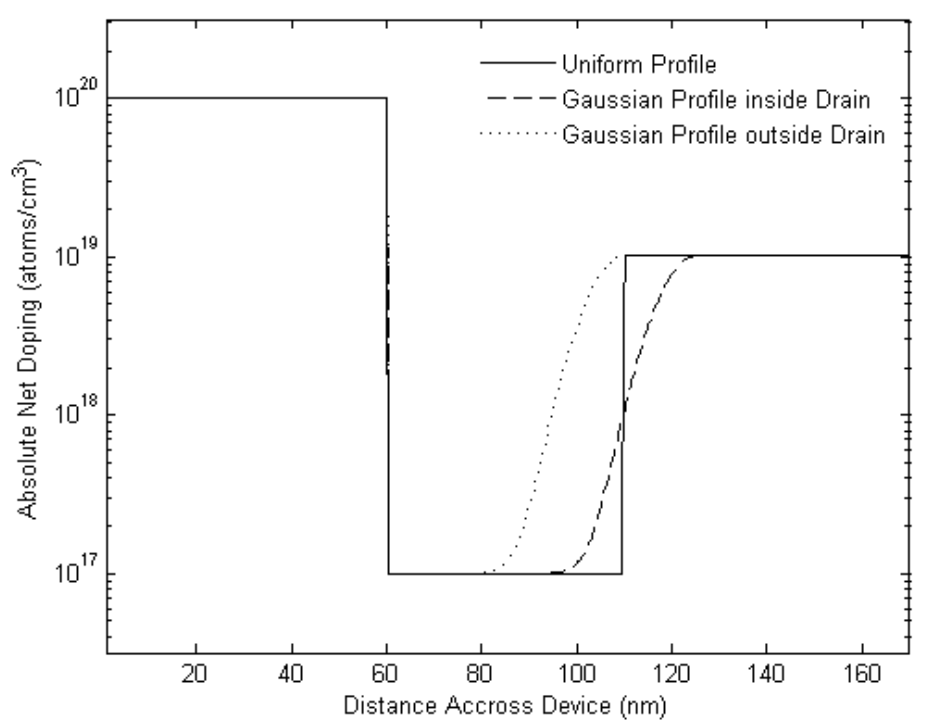

Fig. 2 - Doping profile for drain doping of $1 \times 10^{19}$ atoms $/ \mathrm{cm}^{3}$
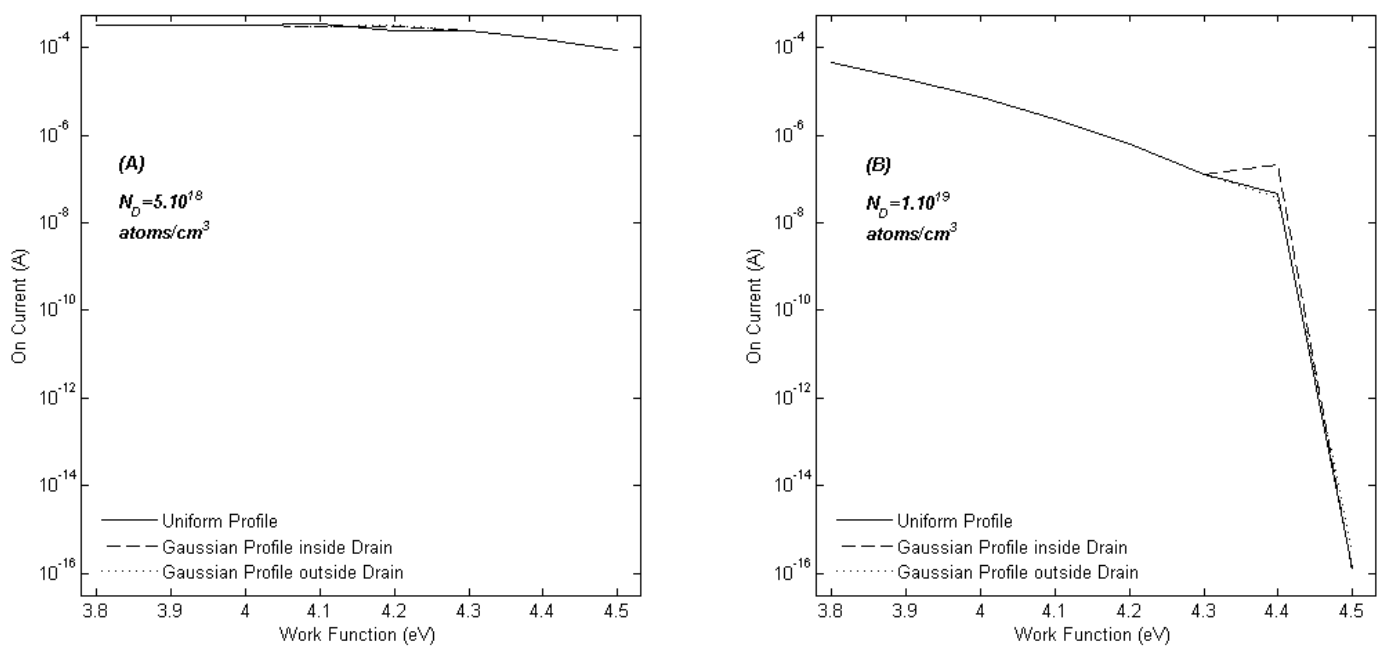

Fig. 3 - The on current in different doping concentrations with the work function

Fig. 3(A) display the on current in logarithmic scale in the doping of $5 \mathrm{e} \times 18$ atoms $/ \mathrm{cm}^{3}$. The three doping profiles are almost the same, while the in-doped Gaussian profile has the highest on current for $4 \mathrm{eV}$ up to $4.3 \mathrm{eV}$ which is around $0.3 \mathrm{~mA}$. While Fig. 3(B) shows that the $1 \times 10^{19}$ atoms $/ \mathrm{cm}^{3}$ doping has the same effect as the previous doping 
concentration but it decreases drastically at work function of $4.4 \mathrm{eV}$. The on current is a result of the BTBT from source to channel which gets effected by the barrier height which is controlled by the work function. While the doping concentration controls the allowed states for the current. When using the Gaussian doping; the depletion width between channel and drain junction increases lowering the tunneling probability causing a decrease in on current. It is for the current. When using the Gaussian doping; the depletion width between channel and drain junction increases lowering the tunneling probability causing a decrease in on current.

Fig. 4(A) the first concentration of $5 \times 10^{18}$ atoms $/ \mathrm{cm}^{3}$ shows how the uniform and Gaussian out-drain doping ambipolar current have the same value of $1 \mathrm{e}-15 \mathrm{~mA}$ and increases with the increasing of the work function and decreases after it reaches 4.4 work function, while the in-drain Gaussian doped increases insignificantly linearly with the work function. The second graph in Fig. 4(B) shows the doping of $1 \times 10^{19}$ atoms $/ \mathrm{cm}^{3}$ has the same effect where the out-drain Gaussian doped and the uniformly doped has the same value of 1e-6 mA while the in-drain Gaussian doped has a value of 1e-10 then keeps decreasing with the increase of the work function. The band to band tunneling in the drain-channel junction is minimized in a TFET by reducing the drain doping that's why the lowest value of the ambipolar current is at the lowest doping concentration [4]. The height of the barrier is controlled by the work function of the metal and the nature of the metal- semiconductor interface. And by increasing the work function the barrier increases making the BTBT from drain to channel harder.
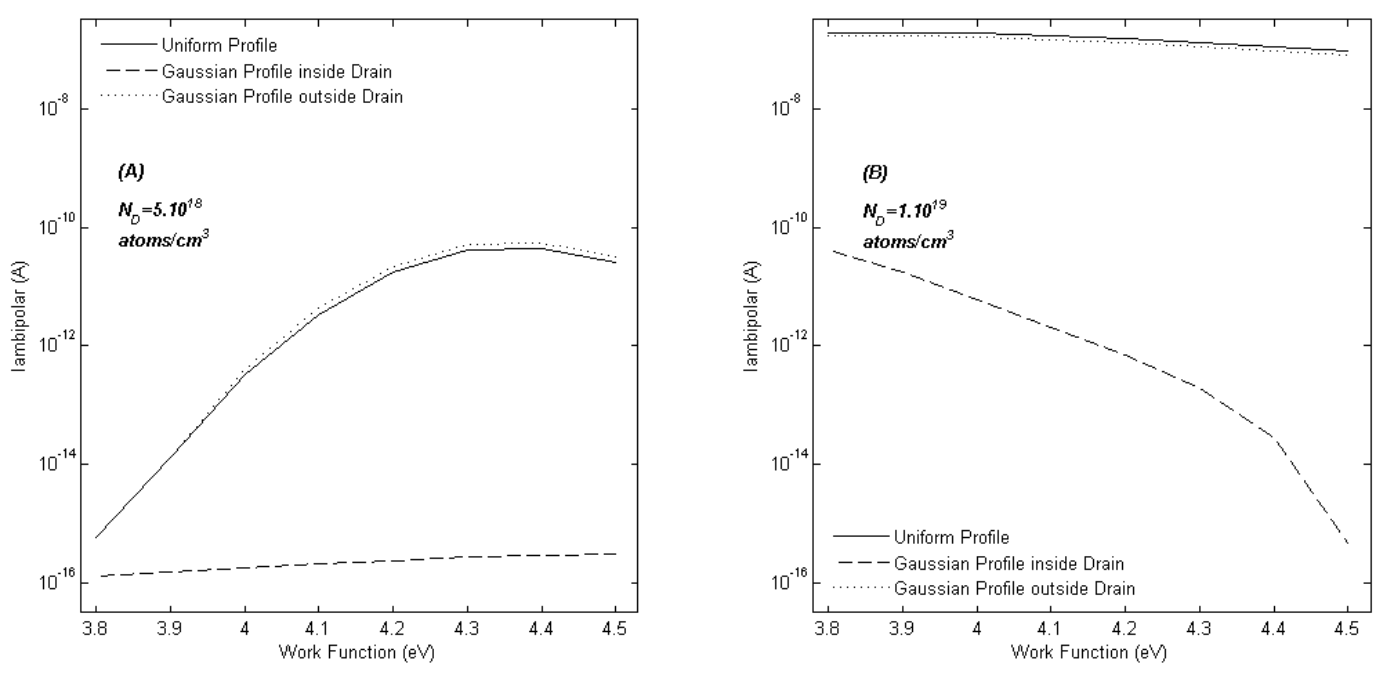

Fig. 4 - The figures above displays the ambipolar current and work function in different doping

At Fig. 5(A) the subthreshold swing in linear scale. In the first doping of $5 \times 10^{18}$ atoms $/ \mathrm{cm}^{3}$ the uniform, Gaussian in- drain and Gaussian out-drain distributions has almost a SS value near $100 \mathrm{mv} / \mathrm{decade}$. In Fig. 5(B) the second doping of $1 \times 10^{19}$ atoms $/ \mathrm{cm}^{3}$ gives the lowest SS of decreasing value from $60 \mathrm{mV} /$ decade until the work function of 4.4 . The average SS of symmetric TFET as in Eq. (2),

$$
S S=\frac{\left(V_{\text {ox }}-V_{\text {off }}\right)}{\left(\log I_{\text {ox }}-\log I_{\text {orf }}\right)}
$$

where $V_{\text {on }}$ is taken to be one volt and $V_{\text {off }}$ is the voltage where the current starts to increase [4,5]. The off-current $I_{\text {off }}$ is defined as the drain current when gate voltage is $\mathrm{V}_{\text {off }}$ and $I_{\text {on }}$ is the drain current at one volt. The subthreshold voltage decreases linearly while increasing the work function, as the whole IV curve shifts toward right as $V_{\text {off }}$ increases, lowering the difference between $\mathrm{I}_{\mathrm{on}}$ and $\mathrm{I}_{\mathrm{off}}$ as shown from the decrease of on/off ratio in Fig. 6 [5]. 

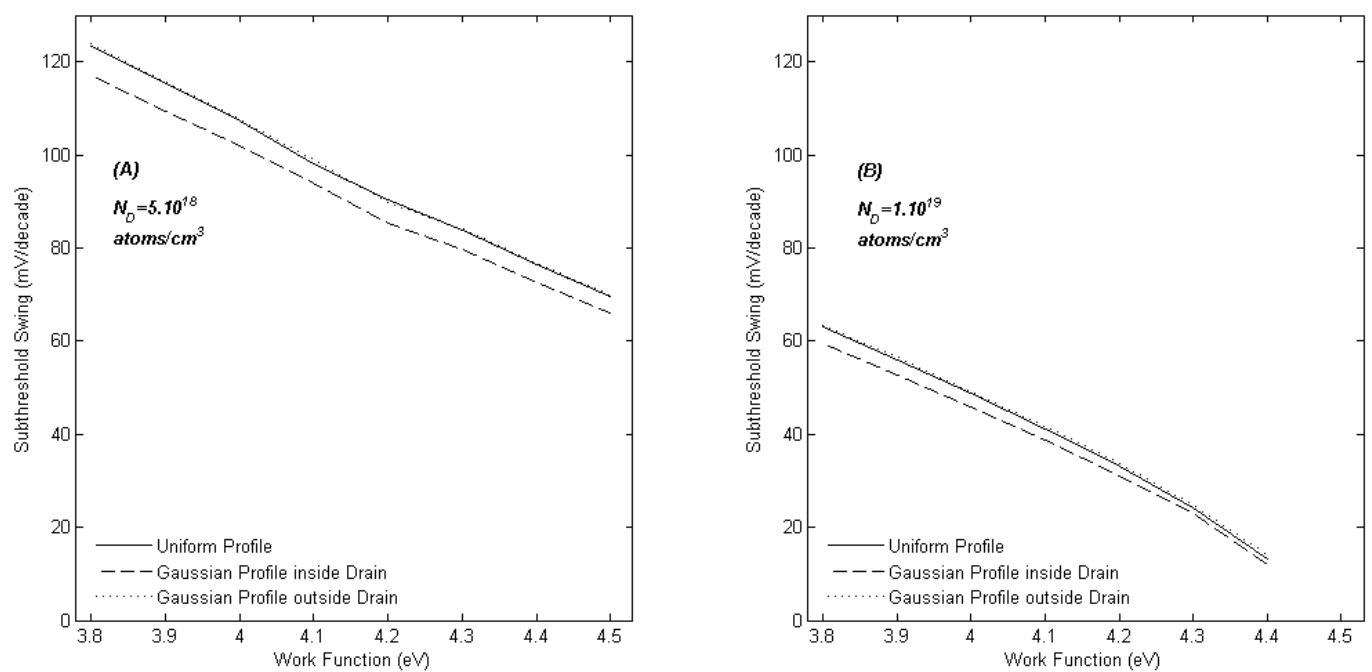

Fig. 5 - The subthreshold swing against the work function

In Fig. 6(A) the ON /OFF current ratio of the Gaussian in-drain doping is the highest value of $1 \times 10^{13}$ due to the low $I_{O F F}$. The Gaussian out-drain and uniform distributions has the value of $1 \times 10^{12}$ then all decrease for the decreasing of the on current at concentration of $5 \times 10^{18}$ atoms $/ \mathrm{cm}^{3}$. In Fig. $6(B)$ that represents the $1 \times 10^{19}$ atoms $/ \mathrm{cm}^{3} \mathrm{doping}$ where the in-drain Gaussian doped has the highest value of $1 \times 10^{10}$ while the uniformly doped drain and Gaussian doped outdrain has the same value of $1 \times 10^{11}$ the decreases abruptly after the work function of 4.4. This sudden decrease is accounted to the way the ON current is defined as the current at one volt, which decreases due to the increase of the gate work function which shifts the whole transfer characteristics to the right. So as the ON current is decreased and the ON/OFF ratio dropped accordingly. Taking in consideration the drain doping increase, which affects more the ON current, the effect of characteristics shift becomes more apparent. When using Gaussian doping the off current decreases causing a higher $I_{\text {On }} / I_{\text {OFF }}$ ratio while the increase of the work function will affect the BTBT in source, so the ratio decreases. After comparing the values, we found that the device that functions at a doping of $5 \times 10^{18}$ and a work function of 4.2 is optimum. And compering that to the conventional TFET shown in Fig. 7.
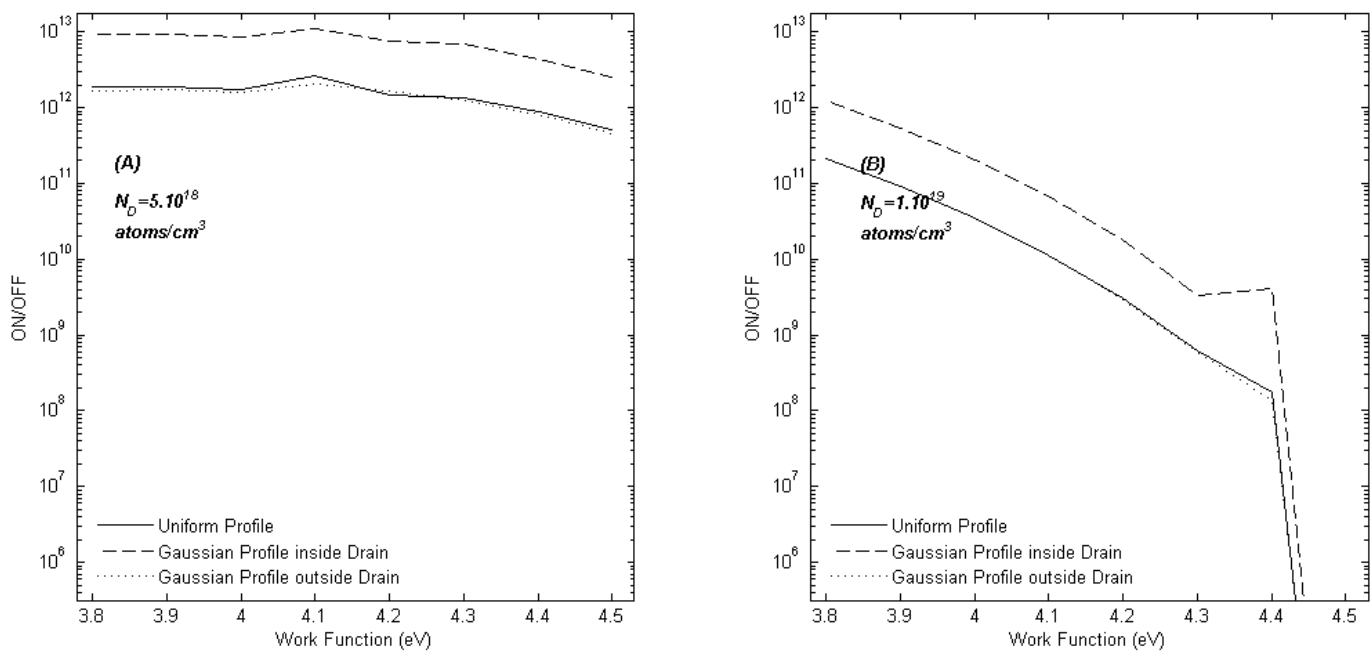

Fig. 6 - The ON /OFF current ratio in logarithmic scale with the work function 
Table 2 - Conventional vs. optimum device

\begin{tabular}{lll}
\hline The device parameter & Conventional device & Optimized device \\
\hline Source Doping $\left(\mathrm{N}_{\mathrm{A}}\right)$ & Uniform & Gaussian \\
Drain Doping $\left(\mathrm{N}_{\mathrm{D}}\right)$ & $1 \times 10^{20}$ atoms $/ \mathrm{cm}^{3}$ & $1 \times 10^{20}$ atoms $/ \mathrm{cm}^{3}$ \\
Channel Doping $\left(\mathrm{N}_{\mathrm{A}}\right.$ or $\left.\mathrm{N}_{\mathrm{D}}\right)$ & $1 \times 10^{19}$ atoms $/ \mathrm{cm}^{3}$ & $5 \times 10^{18}$ atoms $/ \mathrm{cm}^{3}$ \\
Source Length & $1 \times 10^{17}$ atoms $/ \mathrm{cm}^{3}$ & $1 \times 10^{17}$ atoms $/ \mathrm{cm}^{3}$ \\
Drain Length & $50 \mathrm{~nm}$ & $50 \mathrm{~nm}$ \\
Channel Length $(\mathrm{L})$ & $3 \mathrm{~nm}$ & $3 \mathrm{~nm}$ \\
Gate Oxide Thickness $\left(\mathrm{t}_{\mathrm{ox}}\right)$ & $10 \mathrm{~nm}$ & $10 \mathrm{~nm}$ \\
Silicon Body Thickness $\left(\mathrm{t}_{\mathrm{si}}\right)$ & $4.5 \mathrm{eV}$ & $4.2 \mathrm{eV}$ \\
Gate Work Function $\left(\Phi_{\mathrm{m}}\right)$ & Uniform & Gaussian inside Drain \\
\hline
\end{tabular}

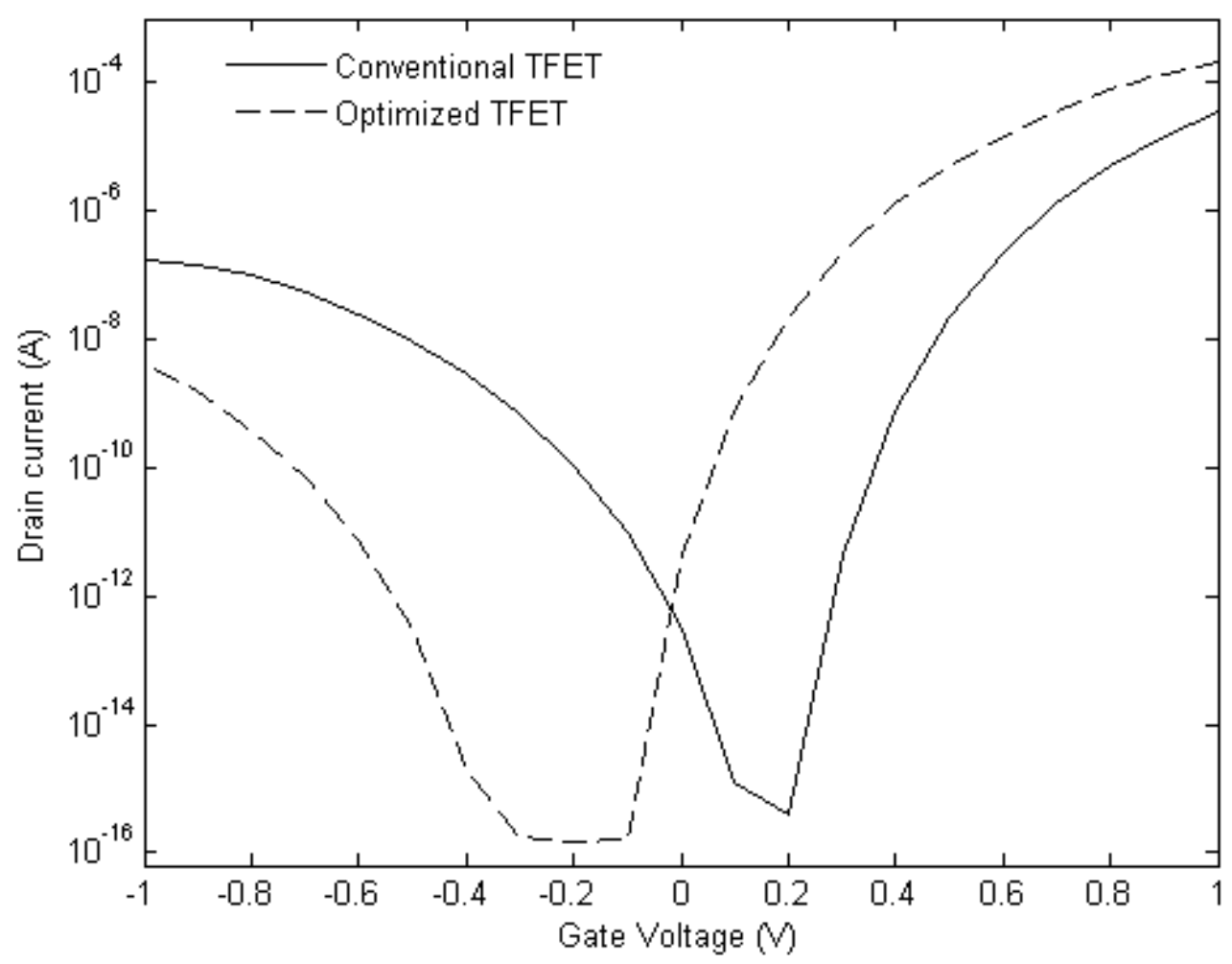

Fig. 7 - Conventional and optimized TFET I-V characteristics

\section{Conclusion}

In this paper, the effect of the gate work function and the doping profile terminating within and outside the drain has been studied. It observed that the optimum characteristics can be achieved with the doping concentration of $5 \times 10^{18} \mathrm{~cm}^{-3}$ and gate work function of $4.2 \mathrm{eV}$ with Gaussian profile inside the drain. Moreover, it observed that when the work function increased from $3.8 \mathrm{eV}$ To $4.5 \mathrm{eV}$ the $\mathrm{I}_{\text {on }} / \mathrm{I}_{\text {off }}$ decreased from $9.17 \times 10^{12}$ to $2.5 \times 10^{12}$, and the subthreshold swing changed from $117.1 \mathrm{mV} /$ decade to $66 \mathrm{mV} /$ decade $\mathrm{mV} /$ decade with Gaussian profile inside the drain.

\section{References}

[1] ZA, N.F., Ahmad, I., Ker, P.J. and Menon, P.S., 2015. Modelling and Characterization of a 14 nm Planar p-Type MOSFET Device. International Journal of Integrated Engineering, 7(3).

[2] bin Wan, W.M.E.A., Ruslan, S.H. and Soh, M.J.C., 2017. Design of Low Power CMOS Bioamplifier in $250 \mathrm{~nm}$ and $90 \mathrm{~nm}$ Technology Node. International Journal of Integrated Engineering, 9(4). 
[3] Saurabh, S., \& Kumar, M. J. (2017). Fundamentals of tunnel field-effect transistors. Boca Raton: CRC Press, Taylor \& Francis Group.

[4] Turkane, S., \& Kureshi, A. K. (2016). Review of Tunnel Field Effect Transistor (TFET). International Journal of Applied Engineering Research, 11(7), 4922-4929.

[5] Ramaswamy, S., \& Kumar, M. J. (2017). Double gate symmetric tunnel FET: investigation and analysis. IET Circuits, Devices \& Systems, 11(4), 365-370.

[6] Choi, W. Y., Park, B. G., Lee, J. D., \& Liu, T. J. K. (2007). Tunneling Field-Effect Transistors (TFETs) With Subthreshold Swing (SS) Less Than $60 \mathrm{mV} / \mathrm{dec}$. IEEE Electron Device Letters, 28(8), 743-745.

[7] Cao, W., Sarkar, D., Khatami, Y., Kang, J., \& Banerjee, K. (2014). Subthreshold-swing physics of tunnel fieldeffect transistors. AIP Advances, 4(6) 067141

[8] Chen, Y. N., Chen, C.J., Fan, M. L., Hu, V., Su, P., \& Chuang, C. T. (2015). Impacts of Work Function Variation and Line-Edge Roughness on TFET and FinFET Devices and 32-Bit CLA Circuits. Journal of Low Power Electronics and Applications, 5(2), 101-115.

[9] Elgamal, Muhammad, and Mostafa Fedawy. "Optimizing Gate-on-Source Overlapped TFET Device Parameters by Changing Gate Differential Work Function and Overlap Dielectric." 2019 International Conference on Innovative Trends in Computer Engineering (ITCE). IEEE, 2019.

[10] Elnaggar, Mohamed, Ahmed Shaker, and Mostafa Fedawy. A comprehensive investigation of TFETs with semiconducting silicide source: impact of gate drain underlap and interface traps.Semiconductor Science and Technology 34.4 (2019): 045015.

[11] Elnaggar, Mohamed, Ahmed Shaker, and Mostafa Fedawy. "Modified Hetero-Gate-Dielectric TFET for Improved Analog and Digital Performance." 2018 13th International Conference on Computer Engineering and Systems (ICCES). IEEE, 2018. 